\title{
Lymph Node Dissection in Resectable Advanced Gastric Cancer
}

\author{
Wobbe O. de Steur $^{\mathrm{a}} \quad$ Johan L. Dikken ${ }^{\mathrm{a}}$ b Henk H. Hartgrink ${ }^{\mathrm{a}}$ \\ ${ }^{a}$ Department of Surgery, Leiden University Medical Center, Leiden, and ${ }^{b}$ Department of Surgery, \\ Medical Center Haaglanden, The Hague, The Netherlands
}

\section{Key Words}

Stomach neoplasms · Surgery $\cdot$ Lymph node dissection

\begin{abstract}
The extent of surgery for gastric cancer has been debated since Billroth performed his first gastrectomy in 1881. This review gives an overview of the available literature on the extent of gastrectomy and lymphadenectomy for advanced resectable gastric cancer. Subtotal gastrectomy is associated with lower morbidity and mortality compared with total gastrectomy, without compromising long-term survival. However, a positive resection margin decreases the chance of curation. Frozen section examination may prevent this. For poorly differentiated singlet ring cell tumors, there may be an argument to perform a total gastrectomy in all cases. In 1981, the Japanese Research Society for the Study of Gastric Cancer provided guidelines for the standardization of surgical treatment and pathological evaluation of gastric cancer. Since then, D2 lymph node dissections have become the standard of care in Japan. Because of the superior stage-specific survival rates in Japan, a D2 dissection was evaluated in several Western randomized controlled trials, but no survival benefit was found for a D2 over a D1 dissection. This might be explained by the increased mortality in the D2 dissection groups which might be the result of a standard pancreaticosplenectomy and low experience with D2 dissections. Add-
\end{abstract}

ing the removal of the para-aortic nodes to a D2 dissection does not further improve survival. The removal of lymph node stations 10 and 11 by splenectomy showed an increased morbidity, no survival benefit, and a very poor prognosis if lymph nodes were affected. Therefore, pancreaticosplenectomy should only be performed in cases of tumor invasion into these organs. A D2 dissection without routine splenectomy and pancreatic tail resection in experienced hands should be considered standard of care for advanced resectable gastric cancer, both in Asian and in Western patients. Centralization and auditing may further improve outcomes after gastrectomy.

Copyright $\odot 2013$ S. Karger AG, Basel

\section{Introduction}

Gastric cancer is the second most frequent cause of cancer death worldwide, affecting approximately one million new individuals each year [1]. Incidence rates vary between countries, with the highest incidence rates in Japan, Korea, and South America, and the lowest rates in Europe, North America, and South and West Asia [2]. Whereas gastric cancers are often detected at a late stage with a corresponding poor prognosis in Western countries, gastric cancers are detected at a much earlier stage in Japan due to mass screening programs

\section{KARGER}

E-Mail karger@karger.com

www.karger.com/dsu
(C) 2013 S. Karger AG, Basel

0253-4886/13/0302-0096\$38.00/0
W.O. de Steur, MD

Department of Surgery, K6-50

Leiden University Medical Center

PO Box 9600, NL-2300 RC Leiden (The Netherlands)

E-Mail w.o.de_steur@lumc.nl 
[3]. This has been mentioned as one of the reasons for superior survival rates in Asian countries compared to Western countries [4]. Surgery is the only curative intervention for patients with advanced (at least submucosal) gastric cancer, while endoscopic (sub)mucosal dissection is frequently used for early gastric cancer in Asia. During the last decade, additional treatment regimens such as chemotherapy and chemoradiotherapy have shown improved survival rates for advanced gastric cancer $[5,6]$. Over the past 30 years, the extent of lymph node dissection has been the subject of an extensive debate worldwide. In Asian countries, extended lymphadenectomy has been a standard procedure since the guidelines were established in 1981 by the Japanese Research Society for the Study of Gastric Cancer (JRSGC), while it has only been recently that Western countries have incorporated extended lymph node dissection into treatment guidelines $[7,8]$. This difference might also be an explanation for the better survival rates in Eastern countries. The purpose of the current review is to give an overview of the available literature on the extent of gastrectomy and lymphadenectomy in advanced resectable gastric cancer.

\section{Total versus Subtotal Gastric Resection}

A total gastrectomy is the therapy of choice for tumors of the proximal or middle stomach [8]. For distal gastric cancers, both a distal and a total gastrectomy can be performed. Several studies have investigated the relation between the extent of resection and morbidity and mortality. In a Norwegian randomized/observational study with 1,010 patients, there was a lower morbidity rate for subtotal gastrectomy ( 28 vs. $38 \%$ ) versus total gastrectomy [9]. Comparable results were found in an observational German study (morbidity 23 vs. 47\%) [10]. In a French study, no difference in 5-year survival was found between a total and subtotal gastric resection [11]. This was confirmed 10 years later in an Italian study [12]. In the Dutch Gastric Cancer Trial (DGCT) and the Medical Research Council (MRC) trial, hospital mortality in the D1 and D2 groups was significantly lower after a subtotal gastrectomy (3 and 7\%, respectively) compared to a total gastrectomy (5 and $14 \%$, respectively) [13-15].

Although a subtotal gastrectomy for distal gastric cancer is associated with less morbidity with an equal longterm survival, the resection line should never be compromised [16]. In the DGCT, $6 \%$ of all patients had microscopic resection line involvement, which was associated with increasing $\mathrm{T}$ stage, $\mathrm{N}$ stage, tumor location, tumor differentiation, and poor survival. This indicates the necessity of per operative frozen section examination, especially for patients with poorly differentiated singlet ring cell tumors. In this context, there is an argument for performing a total gastrectomy in all patients with poor tumor differentiation.

\section{Lymph Node Anatomy and Lymph Node Dissection in Gastric Cancer}

As the primary tumor penetrates more deeply through the wall of the stomach, the risk of lymph node metastases increases. In the JRSGC guidelines, 16 different lymph node stations surrounding the stomach are defined (fig. 1) [17]. These are further divided into four groups, each group further away from the primary tumor site. In a D1 dissection, the stomach (total or distal) plus the perigastric lymph nodes are removed (i.e. stations 1-6). For a D2 dissection, additional removal of the nodes along the left gastric, the common hepatic, the splenic, and the left hepatoduodenal artery (i.e. stations 7-11) is performed. With a D3 and D4 dissection, an even more extended lymphadenectomy is performed, including posterior hepatoduodenal and para-aortic nodes (stations 12 and 16). Minor modifications from this schedule occur depending on the location of the tumor [17].

When Billroth performed his first gastric resection in 1881 , he actually also removed some enlarged nodes. His patient died 14 months after the operation due to recurrent disease. Since then, the extent of lymph node dissection has been a topic of debate in the treatment of gastric cancer. Theoretically, removal of a wide range of lymph nodes improves the chances for cure. Such resection, however, could be irrelevant when no lymph nodes are affected, when the cancer has developed into systemic disease, or the dissection increases morbidity and mortality substantially.

In the 1980s it was shown that stage-specific 5-year survival was higher in Japan compared to the USA: stage $\mathrm{I}=91$ versus $50 \%$, stage II $=72$ versus $29 \%$, stage III $=44$ versus $13 \%$, and stage IV $=9$ versus $3 \%$, respectively [18]. One of the reasons for these differences was thought to be the extent of lymph node dissection. In order to prove that the difference in stage-specific survival was due to the extent of lymph node dissection, randomized controlled trials comparing D1 and D2 dissections were set up in Western countries. 


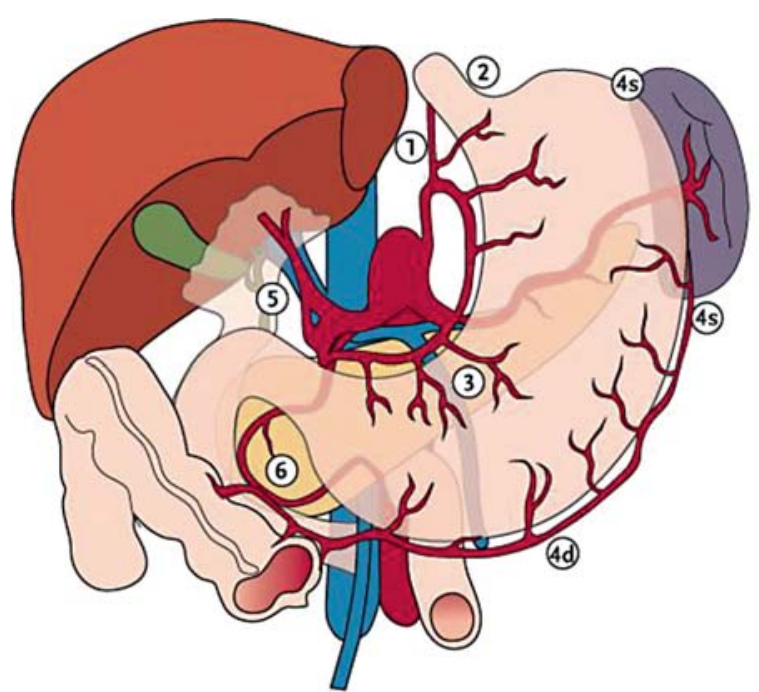

$\mathrm{N} 1$ lymph nodes (perigastric) 1 Right cardiac nodes 2 Left cardiac nodes

3 Nodes along the lesser curvature 4 Nodes along the greater curvature 5 Suprapyloric nodes 6 Infrapyloric nodes

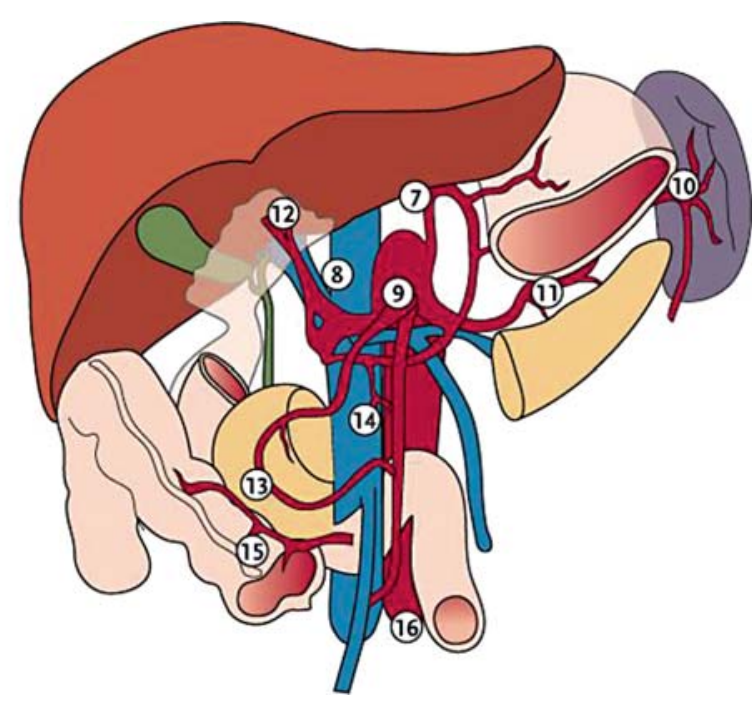

N2 lymph nodes (branches celiac axis) 7 Nodes along root left gastric artery 8 Nodes along common hepatic artery 9 Nodes around celiac axis 10 Nodes at splenic hilum 11 Nodes along splenic artery
N3 lymph nodes

12 Nodes at the hepatoduodenal ligament 13 Retropancreatic (periduodenal) nodes 14 Nodes at the root of the mesentery

N4 lymph nodes

15 Nodes along the middle colic vein 16 Para-aortic nodes

Fig. 1. Lymph node stations as defined by the JRSGC [17]. D1 resection = Removal of the N1 lymph nodes; D2 resection $=$ removal of the $\mathrm{N} 1$ and $\mathrm{N} 2$ lymph nodes.

\section{Randomized Controlled Trials on the Extent of Lymph Node Dissection}

The first trial was started in 1982 in Cape Town, South Africa and was presented by Dent et al. [19] in 1988. A randomized comparison was made for D1 and D2 dissections. In a period of 5 years, 403 patients were evaluated for surgery. All ineligible patients had advanced disease in excess of the protocol stage. Forty-three patients were randomized: 22 in the D1 resection group and 21 in the $\mathrm{D} 2$ resection group (then called $\mathrm{R} 1$ and $\mathrm{R} 2$, respectively). The age in the D2 group was higher and there were more male patients. Patients in the D2 group had a longer operating time, needed more blood transfusions, and had a longer hospital stay. Median follow-up was 3.1 years. No difference in survival was found, while morbidity in the D2 group was higher (table 1). From this study it was concluded a D2 resection did not show a survival benefit over a $\mathrm{D} 1$ resection and that the $\mathrm{D} 2$ resection should only be performed in clinical trials [19].
Robertson et al. [20] presented the second trial in 1994. They performed a randomized controlled trial in Hong Kong, comparing D1 subtotal with D3 total gastrectomy in antral cancer. Fifty-four patients were included in the period 1987-1991. Twenty-five patients underwent a D1 subtotal gastrectomy and 30 patients a D3 total gastrectomy. D3 resection was defined as total gastrectomy and excision of greater and lesser omentum, splenectomy, distal pancreatectomy, and lymph node dissection of the celiac axis and in the hepatoduodenal ligament. D1 resection was defined as subtotal gastrectomy including a $6-\mathrm{cm}$ proximal resection margin, and resection of the lesser and greater omentum. Patient characteristics were comparable between the groups. At 5 years, 7 out of 25 patients (28\%) had died in the D1 group, whereas 14 out of 30 patients $(46 \%)$ had died in the D3 group. The median overall survival was better for the D1 group (1,511 vs. 922 days, respectively). In the D3 group, 1 patient died postoperatively, but no patients died in the D1 group. Morbidity was higher in the D3 group: longer operating time, 
Table 1. Randomized trials for extension of lymphadenectomy

\begin{tabular}{lllcccc}
\hline Study & Accrual & Comparing & Patients, $\mathrm{n}$ & Morbidity, \% & Mortality, \% & 5-Year survival, \% \\
\hline Dent et al. [19] & $1982-1985$ & D1, D2 & 22,21 & 22,43 & 0,0 & 69,67 \\
Robertson et al. [20] & $1987-1991$ & D1, D2 & 25,30 & 0,58 & $0,3.3$ & 45,35 \\
Cuschieri et al. [14] & $1987-1994$ & D1, D2 & 200,200 & 28,46 & $6.5,13$ & 35,33 \\
Bonenkamp et al. [13] & $1989-1993$ & D1, D2 & 380,331 & 25,43 & 4,10 & 45,47 \\
Degiuli et al. [26] & $1998-2005$ & D1, D2 & 133,134 & 18,12 & $3.0,2.2$ & NR \\
Wu et al. [27] & $1993-1999$ & D1, D3 & 110,111 & 7,17 & 0,0 & $53.6,59.5$ \\
Sasako et al. [28] & $1995-2001$ & D2, D4 & 263,260 & 21,28 & $0.8,0.8$ & 69,70 \\
\hline
\end{tabular}

D1 = Limited lymph node dissection; D2 = extended lymph node dissection; D3 = D2+ lymph nodes in the hepatoduodenal ligament; D4 = D2+PAND; NR = not recorded.

more blood loss, more blood transfusions needed, and longer hospital stay ( 8 vs. 16 days). There were 14 cases of subphrenic abscesses in the D3 group. The conclusion of this study was that the routine use of a D3 total gastrectomy in patients with antral gastric cancer was not supported [20].

The MRC performed a large randomized controlled trial in the United Kingdom [14]. A total of 400 patients were randomized to a D1 or D2 lymphadenectomy and underwent a total or subtotal gastrectomy performed by one of 32 participating surgeons in multiple hospitals. A D1 dissection was defined as removal of lymph nodes within $3 \mathrm{~cm}$ of the tumor en bloc with the greater omentum. In a D2 resection for antral tumors, the omental bur$\mathrm{sa}$, the hepatoduodenal, and retroduodenal lymph nodes were resected. For middle and upper third lesions, splenic hilar nodes and retropancreatic nodes were removed by distal hemipancreaticosplenectomy. In 7 years, 737 patients were registered with proven gastric cancer, of which 400 were eligible for the study.

After 5 years, no difference in overall survival ( 35 vs. $33 \%$ for D1 and D2, respectively) and recurrence-free survival were found. In a multivariate analysis, the patients who underwent a spleen- and pancreas-sparing D2 dissection had a better survival rate than the corresponding D1 group. The authors concluded that there was no benefit from a D2 resection, but when sparing the pancreas and spleen there can be an advantage from a D2 dissection compared to a D1 dissection [14].

Nearly simultaneously, a multicenter randomized controlled trial was performed in the Netherlands, which accrued between 1989 and 1993 [13]. In the DGCT, 1,078 patients with gastric cancer were randomized to undergo either a D1 or a D2 lymph node dissection. The study was performed at 80 Dutch hospitals. The surgical treatment in the $\mathrm{D} 2$ group was performed by 11 specially trained supervising surgeons. D1 dissections were performed without supervision as this was the standard procedure in the Netherlands. A total of 711 patients with curable gastric cancer were eligible for curative surgery. Mortality was higher in the D2 group ( 10 vs. $4 \%$, respectively) as well as morbidity ( 43 vs. $25 \%$, respectively). Mean hospital stay was significantly longer for D2 dissections (22 vs. 18 days) and the reoperation rate in the D2 group was higher (16 vs. $8 \%$, respectively). The main causes for reoperation were intra-abdominal abscesses and pulmonary problems. The 5-year survival rate was not found to be different between the $\mathrm{D} 1$ and $\mathrm{D} 2$ dissection groups (45 and $47 \%$, respectively) $[13,21]$.

In 2004 the long-term results of the DGCT were published. After 11 years, there was no significant difference in overall survival (D1 $=30 \%$ vs. $\mathrm{D} 2=35 \%)$. In a subgroup analysis it was found that only patients with $\mathrm{N} 2$ disease might benefit from a D2 resection. The extent of lymph node dissection directly correlated with morbidity and mortality, but the highest risk ratio for morbidity and mortality were pancreatectomy and splenectomy. It was concluded that if mortality and morbidity could be avoided, a D2 resection may be of benefit [22]. Furthermore, analysis of the subgroup that did not undergo pancreaticosplenectomy showed a significant survival advantage for those who had a D2 lymph node dissection (11-year survival $33 \%$ for D1 and $47 \%$ for D2) [23]. In the 15-year follow-up analysis, it was shown that D2 lymphadenectomy was associated with lower locoregional recurrence and gastric cancer-related death rates compared to D1 surgery. The final conclusion was that with safer spleenpreserving D2 dissections, when applied in experienced 
centers, a D2 lymphadenectomy is the recommended surgical approach for patients with resectable gastric cancer [24].

A Cochrane review of these first four randomized trials showed a significantly increased mortality after a D2 dissection (risk ratio: 2.23 , 95\% CI: $1.45-3.45$ ) without a benefit in survival [hazard ratio (HR): 0.95, 95\% CI: $0.83-$ 1.09] [25].

The high postoperative mortality rates after a D2 lymphadenectomy in the Dutch and MRC trials were criticized as both studies were performed in a large number of hospitals without proper training for D2 dissections. Therefore, a randomized D1-D2 study was repeated in Italy after the participating surgeons received specialized training.

In a period of 7 years, 267 patients were randomized in only five hospitals. A D1 dissection was defined as tumor removal with the perigastric lymph nodes according to the JRSGC. A D2 gastrectomy was defined as total gastrectomy with or without a routine splenectomy or pancreatic tail resection. The overall complication rate for the D1 group was 12 versus $17.9 \%$ for the D2 group (difference not significant). Postoperative mortality was $3.0 \%$ for the $\mathrm{D} 1$ dissection group and 2.2\% for the $\mathrm{D} 2$ dissection group. It was shown that a D2 resection for gastric cancer, preserving the pancreas, has equal morbidity and mortality rates as a gastrectomy with D1 lymphadenectomy. We have to wait to see, however, whether there is a survival benefit for D2 dissections in this study group [26].

In order to find out whether more extended lymph node dissections were beneficial for Eastern patients, two randomized trials were performed. The first one from Taiwan, which started accrual in 1993, was a single institution randomized trial comparing D1 and D3 resection in gastric cancer. In a period of 6 years, 335 patients were registered, of which 221 patients were randomized to a D1 or D3 resection. The primary endpoints were 5-year overall survival and 5-year disease-specific survival. All patients were operated by three well-trained ( $>25$ independent D3 resections) surgeons. A D1 resection was defined as tumor removal with a $3-\mathrm{cm}$ margin for superficial well-differentiated tumors and lymph node resection along the greater and lesser curvature. A D3 resection was defined as tumor removal with a subtotal or total gastric resection with dissection of the lymph nodes around the blood vessels supplying the stomach that arise from the celiac axis, lymph nodes in the hepatoduodenal ligament, retropancreatic region, and lymph nodes surrounding the superior mesenteric vein. Baseline characteristics of both groups were the same. A total of 215 patients under- went a R0 resection. Overall survival was significantly better for the D3 group (59.5\%) compared to the D1 group (53.6\%; $\mathrm{p}=0.041)$. Disease-specific survival was $54.3 \%$ for the D3 group versus $49.5 \%$ for the D1 group (per protocol analysis). Although morbidity was higher after a D3 dissection, there was no mortality in either group. Therefore, D3 resection, when performed by welltrained surgeons, offers a survival benefit over D1 dissection in patients with gastric cancer [27].

The second study is from Japan. This multi-institutional randomized controlled trial by the Japanese Clinical Oncology Group (JCOG 9501) was developed to determine whether the addition of systematic para-aortic lymph node dissection (PAND) to standard gastrectomy with D2 lymphadenectomy improved survival rates among patients with curable gastric cancer. Between 1995 and 2001, 523 patients were randomized to a D2 resection $(\mathrm{n}=263)$ and a D2+PAND $(\mathrm{n}=260)$. The study was performed at 24 hospitals. Participating surgeons had performed at least 100 gastrectomies with a D2 dissection, or the operation was carried out in a hospital where more than 80 gastrectomies are performed per year. The postoperative complication rate was $20.9 \%$ for the D2 group and $28.1 \%$ for the D2+PAND group. There were no significant differences between the two groups in frequencies of anastomotic leakage, pancreatic fistula, abdominal abscess, pneumonia, or death from any cause within 30 days after surgery. There was a longer operation time (median: $63 \mathrm{~min}$ ) and more blood loss $(230 \mathrm{ml})$ in the $\mathrm{D} 2+\mathrm{PAND}$ group. The 5 -year survival rate was not significantly different: $69.2 \%$ for the D2 group and $70.3 \%$ for the D2+PAND group. There also were no significant differences in recurrence-free survival between the two groups. Therefore, a more extended dissection (D2+PAND) did not improve the survival rate in curable gastric cancer [28].

\section{Maruyama Index}

In order to make lymph node dissections more tailormade, Prof. Maruyama introduced the Maruyama Index (MI) of unresected disease after meticulously studying all resected lymph node stations in 3,843 patients $[18,29]$. Based on seven input variables (age, sex, Borrmann type, tumor size, tumor position, and histology), the likelihood for nodal involvement of each lymph node station (fig. 1) can be calculated by the Maruyama Computer Program.

The MI of unresected disease has later been defined as the sum of regional nodal disease percentages for stations 
(1-12) not removed by the surgeon. It was developed to predict survival of patients with gastric cancer after surgery based on patient and tumor characteristics combined with information on the removed lymph node stations. This index was calculated for 648 patients in the DGCT. An MI of less than 5 was associated with a significantly higher survival and a reduced relapse risk compared with patients who scored 5 or more [30]. This index also proved to be a significant predictor of survival on both univariate and multivariate analyses in the Intergroup 0116 trial [31]. Because of its complexity for use in the operating room, the MI is not frequently used during surgery in Western countries.

\section{Removing Lymph Node Stations 10 and 11 and Spleen}

Another issue is whether removal of the spleen offers a benefit in survival. On one hand, the removal of the spleen may be beneficial in order to adequately remove lymph node stations 10 and 11 and thereby improve survival. On the other hand, removal of the spleen may lead to increased morbidity due to pancreatic fistula, intraabdominal abscesses, and anastomotic leakage.

In the DGCT, removal of the spleen and pancreatic tail led to a strong increase in morbidity and mortality [13]. The most severe complications such as pancreatic fistula (3 vs. $1 \%$ ), intra-abdominal abscesses (17 vs. $8 \%$ ), and anastomotic leakage (9 vs. 4\%) occurred significantly more in the $\mathrm{D} 2$ dissection group.

In the DGCT, lymph node stations 10 and 11 were removed in 112 and 124 patients, respectively. In the group of 18 patients with metastasis in lymph node station 10 , survival after 11 years of follow-up was $11 \%$. In the group of 24 patients with metastasis in station 11, the 11-year survival was only $8 \%$. In patients without metastasis in lymph node stations 10 and 11, the 11-year survival was 27 and $35 \%$, respectively [22].

In a later subgroup analysis of patients who did not undergo a pancreaticosplenectomy in the DGCT, a significant survival advantage for those who had a D2 lymph node dissection was found (11-year survival of $33 \%$ for $\mathrm{D} 1$ and $47 \%$ for $\mathrm{D} 2, \mathrm{p}=0.018$ ) [23]. Thus, there may be a rationale to perform a $\mathrm{D} 2$ dissection if morbidity and mortality can be prevented.

To date, three randomized controlled trials have been performed that have evaluated the effect of splenectomy for gastric cancer. In a Japanese study, the lymph nodes along the splenic artery were removed in a pancreas-pre-

Lymph Node Dissection in Advanced Resectable Gastric Cancer
Table 2. Randomized trials on splenectomy for curable gastric cancer

\begin{tabular}{llllll}
\hline Study & $\begin{array}{l}\text { Patients } \\
\mathrm{n}\end{array}$ & $\begin{array}{l}\text { Surgical } \\
\text { complica- } \\
\text { tion rate, \% }\end{array}$ & $\begin{array}{l}\text { Mortality } \\
\%\end{array}$ & $\begin{array}{l}\text { Overall } \\
\text { survival } \\
5 \text {-year, \% }\end{array}$ & $\mathrm{p}$ \\
\hline $\begin{array}{l}\text { Furakawa } \\
\text { et al. [32] }\end{array}$ & $\mathrm{S}-=55$ & $13^{\mathrm{a}}$ & $\mathrm{NR}$ & 76.7 & $\mathrm{NS}^{\mathrm{b}}$ \\
\hline Csendes & $\mathrm{S}-=55$ & $16^{\mathrm{a}}$ & $\mathrm{NR}$ & 80.0 & \\
et al. [33] & $\mathrm{S}+=90$ & $\mathrm{NR}$ & 3.1 & 36 & $\mathrm{NS}$ \\
\hline Yu et al. [34] & $\mathrm{S}-=103$ & 8.7 & 4.4 & 42 & \\
& $\mathrm{~S}+=104$ & 15.4 & 1.0 & 48.8 & $\mathrm{NS}$ \\
& & & & 54.8 & \\
\hline
\end{tabular}

S- = Without splenectomy; + + = with splenectomy; NR = not reported; NS = not significant.

a Pancreatic fistula and anastomotic leakage.

b Not significant for all items.

serving way. A total of 110 patients (55 in each group) were randomized for total gastrectomy with or without splenectomy. No significant differences were found in 5- and 10-year survival (table 2). Although anastomotic leakage was equal in both groups (3.6\%), the number of pancreatic fistula was significantly higher in the splenectomy group (9.1 vs. $14.5 \%$ ) [32].

In a Chilean trial, 187 patients underwent total gastrectomy with or without splenectomy (90 with and 97 without). There was also no difference found in survival in this study. Perioperative mortality was 4.4 and $3.1 \%$ for patients with or without splenectomy, respectively. Septic complications like pulmonary problems and subphrenic abscesses occurred significantly more often in the splenectomy resection group [33].

In a Korean trial, 207 patients were randomized for gastrectomy with or without splenectomy. Although 5 -year survival was higher in the splenectomy group, this difference did not reach statistical significance ( 48.8 vs. $54.8 \%)$. Surgical complications occurred more frequently in the splenectomy group (15.4 vs. $8.7 \%$ ), but this difference also did not reach statistical significance [34].

A meta-analysis on perioperative survival with the data from these randomized trials showed no statistically significant difference (OR: 1.59, 95\% CI: 0.44-5.79) [35]. A large randomized trial to evaluate the use of splenectomy in gastric cancer is currently underway in Japan [36].

A systematic review of spleen and pancreas preservation in extended lymphadenectomy for gastric cancer was recently presented by Brar et al. [35]. Besides the random- 
ized trials, 37 articles with 6,354 patients were included in this review. From this analysis it was concluded that despite the concern about the increased morbidity and mortality of splenectomy and distal pancreatectomy during extended lymphadenectomy for gastric cancer as demonstrated in some studies, more contemporary studies demonstrate that this procedure can still be done safely with low short-term morbidity and mortality for patients undergoing curative surgery for gastric cancer. However, in the studied randomized controlled trials, no difference in 5-year survival was found [35].

As far as reported, most of these studies have in common that prognosis for patients with affected lymph nodes in the splenic hilus is poor. Taking this into account together with the increased morbidity without survival benefit, splenectomy cannot be advised at this moment unless there is direct invasion into the spleen. The results of the JCOG 0110 trial are awaited with great interest [36].

\section{Hospital Volume and Surgeon Volume}

There is a large difference in the incidence of gastric cancer between Asia and Europe. In Japan there is a screening program for gastric cancer and Japan has a twoto sevenfold higher incidence of gastric cancer compared to European countries. European surgeons are exposed to much less gastric cancer than their Asian counterparts. One of the comments on the European D1-D2 studies was that the cause of the high mortality could be the low exposure the surgeons have for gastric cancer. Performing these randomized trials, it has been shown that highrisk low-volume surgery, like gastric cancer, can improve outcome over a longer period. Increasing surgeon and hospital volume is the key to improvement [37].

In the Western world and Asia there are many studies that have shown the relation between outcome and hospital volume. Increasing the surgeons' volume also contributed to a higher survival rate and lower postoperative mortality [38]. In 2003, Denmark enforced centralization of gastric cancer surgery from 37 to 5 hospitals, which resulted in a significant decrease in postoperative mortality ( $8.2 \%$ in 2003 to $2.4 \%$ in $2008, \mathrm{p}=0.05$ ) and an increase in the number of patients with at least 15 lymph nodes examined (19-67\%) [39]. Centralization of gastric cancer surgery is currently being implemented in the UK, Sweden, Finland, and in almost all regions in the Netherlands.

One of the other key solutions for the improvement of gastric cancer is auditing. With auditing, surgeons can improve their results by learning from their own outcome statistics benchmarked against their peers, which is often referred to as the Hawthorne effect. With auditing it is possible to analyze differences in hospital mortality, the extent of lymph node dissection, and the use of laparoscopic techniques. Auditing has proven its value in rectal cancer treatment in Europe [40], and audits for gastric and esophageal cancer are currently used in Denmark, the UK, and the Netherlands.

\section{Discussion}

The only way to cure patients with advanced gastric cancer is gastrectomy with adequate regional lymph node dissection. In Japan and Korea the standard of care has been a gastrectomy with $\mathrm{D} 2$ lymph node dissection for many years, while it has only recently been implemented as the standard of care in Western countries.

The DGCT and MRC trials did not show any benefit for a D2 dissection after 5 years. This might be explained by inadequacy of pretrial surgical training and the consequent increased morbidity and mortality. Multiple studies have reported a close relation between the number of cases treated in a hospital and outcome in the surgical treatment of cancer [38]. An Italian trial with adequately trained surgeons showed that a D2 dissection can be performed safely in Western countries [26]. In Europe only the long-term follow-up of the DGCT showed a decrease in cancer-related deaths after D2 dissection [24].

The high mortality after D2 dissection in the DGCT and MRC trial has probably offset the possible survival benefit. Subgroup analysis showed a very strong independent association between postoperative death and resection of the spleen and tail of the pancreas. Patients who did not undergo a pancreaticosplenectomy had a significant survival advantage for those who had a D2 lymph node dissection [23].

When postoperative mortality can be avoided, an extended lymph node dissection may be beneficial as shown by Wu et al. [27]. More extended lymph node dissections with the removal of para-aortic lymph nodes, however, show no further benefit in survival [28]. This suggests that if para-aortic lymph nodes are affected, there probably already is systemic disease.

In conclusion, D2 lymph node dissection should be the standard lymph node dissection for advanced curable gastric cancer. The removal of the spleen and pancreatic tail should only be performed in cases with direct tumor invasion into these organs. Centralization and auditing may further improve the outcome for gastrectomy. 


\section{References}

1 Kamangar F, Dores GM, Anderson WF: Patterns of cancer incidence, mortality, and prevalence across five continents: defining priorities to reduce cancerdisparities in different geographic regions of the world. J Clin Oncol 2006;24:2137-2150.

-2 Yamaoka Y, Kato M, Asaka M: Geographic differences in gastric cancer incidence can be explained by differences between Helicobacter pylori strains. Intern Med 2008;47: 1077-1083.

$\checkmark 3$ Hamashima C, Shibuya D, Yamazaki H, et al: The Japanese guidelines for gastric cancer screening. Jpn J Clin Oncol 2008;38:259-267.

4 Hundahl A, Phillips JL, Menck HR, et al: The National Cancer Data Base Report on poor survival of US gastric carcinoma patients treated with gastrectomy: Fifth Edition American Joint Committee on Cancer staging, proximal, and the 'different disease' hypothesis. Cancer 2000;88:921-932.

$\checkmark 5$ Macdonald JS, Smalley SR, Benedetti J, et al: Chemoradiotherapy after surgery compared with surgery alone for adenocarcinoma of the stomach or gastroesophageal junction. N Engl J Med 2001;345:725-730.

-6 Cunningham D, Allum WH, Stenning SP, et al: Perioperative chemotherapy versus surgery alone for resectable gastroesophageal cancer. N Engl J Med 2006;355:11-20.

$>7$ Okines A, Verheij M, Allum W, Cunningham D, Cervantes A, ESMO Guidelines Working Group: Gastric cancer: ESMO Clinical Practice Guidelines for diagnosis, treatment and follow-up. Ann Oncol 2010;21(suppl 5):v50-v54.

8 NCCN: National Comprehensive Cancer Network Clinical Practice Guidelines in Oncology. Gastric Cancer, Version 2, 2011. http://www.nccn.org.

$>9$ Viste A, Haugstvedt T, Eide GE, Soreide O: Postoperative complications and mortality after surgery for gastric cancer. Ann Surg 1988;207:7-12.

-10 Bottcher K, Siewert JR, Roder JD, Busch R, Hermanek P, Meyer HJ: Risiko der chirurgischen Therapie des Magencarcinoms in Deutschland: Ergebnisse der Deutschen Magencarcinom-Studie 1992. Chirurg 1994;65: 298-306.

11 Gouzi JL, Huguier M, Fagniez PL, et al: Total versus subtotal gastrectomy for adenocarcinoma of the gastric antrum. A French prospective controlled study. Ann Surg 1989;209: $162-166$.

-12 Bozzetti F, Marubini E, Bonfanti G, Miceli R, Piano C, Gennari L: Subtotal versus total gastrectomy for gastric cancer: five year survival rates in a multicenter randomized Italian trial. Gastrointestinal Tumor Study Group. Ann Surg 1999;230:170-178.

-13 Bonenkamp JJ, van de Velde CJ, Songun I, et al: Randomised comparison of morbidity after D1 and D2 dissection for gastric cancer in 996 Dutch patients. Lancet 1995;345:745748.
14 Cuschieri A, Weeden S, Fielding J, et al: Patient survival after D1 and D2 resections for gastric cancer: long-term results of the MRC randomized surgical trial. Surgical Co-operative Group. Br J Cancer 1999;79:15221530.

15 Sasako M: Risk factors for surgical treatment in the Dutch Gastric Cancer Trial. Br J Surg 1997;84:1567-1571.

16 Songun I, Bonenkamp JJ, Hermans J, van Krieken JH, van de Velde CJ: Prognostic value of resection-line involvement in patients undergoing curative resections for gastric cancer. Eur J Cancer 1996;32:433-437.

17 Kijitani T: Japanese Research Society for the Study of Gastric Cancer. The general rules for gastric cancer study and pathology. Jap J Surg 1981;11:127-145.

-18 Maruyama K, Okabayashi K, Kinoshita T: Progress in gastric cancer surgery in Japan and its limits of radicality. World J Surg 1987; 11:418-425.

19 Dent DM, Madden MV, Price SK: Randomized comparison of R1 and R2 gastrectomy for gastric carcinoma. Br J Surg 1988;75:110 112.

20 Robertson CS, Chung SC, Woods SD, et al: A prospective randomized trial comparing R1 subtotal gastrectomy with R3 total gastrectomy for antral cancer. Ann Surg 1994;220:176182.

21 Bonenkamp JJ, Hermans J, Sasako M, van de Velde CJ, Dutch Gastric Cancer trial group: Extended lymph-node dissection for gastric cancer. N Engl J Med 1999;340:908-914.

22 Hartgrink HH, van de Velde CJ, Putter H, et al: Extended lymph node dissection for gastric cancer: who may benefit? Final results of the randomized Dutch Gastric Cancer Group Trial. J Clin Oncol 2004;22:2069-2077.

23 Hartgrink HH, Jansen EP, van Grieken NC, van de Velde CI: Gastric cancer - seminar. Lancet 2009;374:477-490.

24 Songun I, Putter H, Meershoek-Klein Kranenbarg E, Sasako M, van de Velde CJ: Surgical treatment of gastric cancer: 15 -year follow-up results of the randomised nationwide Dutch D1D2 trial. Lancet Oncol 2010; 11:439-449.

25 McCulloch P, Nita ME, Kazi H, Gama-Rodrigues J: Extended versus limited lymph nodes dissection technique for adenocarcinoma of the stomach. Cochrane Database Syst Rev 2004;4:CD001964.

26 Degiuli M, Sasako M, Ponti A, Italian Gastric Cancer Study Group: Br J Surg 2010;97:643649.

27 Wu CW, Hsiung CA, Lo SS, et al: Nodal dissection for patients with gastric cancer: a randomized controlled trial. Lancet Oncol 2006; 7:309-315.

28 Sasako M, Sano T, Yamamoto S, et al: D2 lymphadenectomy alone or with para-aortic nodal dissection for gastric cancer. $\mathrm{N}$ Engl J Med 2008;359:453-462.
29 Kampschoer GH, Maruyama K, van de Velde CJ, Sasako M, Kinoshita T, Okabayashi K: Computer analysis in making preoperative decisions: a rational approach to lymph node dissection in gastric cancer patients. Br J Surg 1989;76:905-908.

30 Peeters KC, Hundahl SA, Kranenbarg EK, Hartgrink $\mathrm{HH}$, van de Velde CJ: Low Maruyama index surgery for gastric cancer: blinded re-analysis of the Dutch D1-D2 trial. World J Surg 2005;29:1576-1584.

31 Hundahl SA, Macdonald JS, Benedetti J, Fitzsimmons T, Southwest Oncology Group and the Gastric Intergroup: Surgical treatment variation in a prospective, randomized trial of chemoradiotherapy in gastric cancer: the effect of undertreatment. Ann Surg Oncol 2002;9:278-286.

32 Furakawa $\mathrm{H}$, Hiratsuka M, Ishikawa O, et al: Total gastrectomy with dissection of lymph nodes along the splenic artery: a pancreas preserving method. Ann Surg 2000;7:669-673.

33 Csendes A, Burdiles P, Rojas J, Braghetto I, Diaz JC, Maluenda F: A prospective randomized study comparing D2 total gastrectomy versus D2 total gastrectomy plus splenectomy in 187 patients with gastric carcinoma. Surgery 2002;131:401-407.

34 Yu W, Choi GS, Chung HY: Randomised clinical trial of splenectomy versus splenic preservation in patients with proximal gastric cancer. Br J Surg 2006;93:559-563.

35 Brar SS, Seevaratnam R, Cardoso R, Law C, Helyer L, Coburn N: A systematic review of spleen and pancreas preservation in extended lymphadenectomy for gastric cancer. Gastric Cancer 2012;15(suppl 1):S89-S99.

36 Sano T, Yamamoto S, Sasako M: Randomised controlled trial to evaluate splenectomy in total gastrectomy fgor gastric adenocarcinoma: Japan Clinical Oncology Group Study JCOG 0110-MF. Jpn J Clin Oncol 2002;32:363-364.

37 Krijnen P, den Dulk M, Meershoek-Klein Kranenbarg E, Jansen-Lansheer ML, van de Velde CJ: Improved survival after resectable non-cardia gastric cancer in the Netherlands: the importance of surgical training and quality control. Eur J Surg Oncol 2009;35:715720 .

38 Birkmeyer JD, Siewers AE, Finlayson EV, et al: Hospital volume and surgical mortality in the United States. N Engl J Med 2002;346: 1128-1137.

-39 Jensen LS, Nielsen H, Mortensen PB, Pilegaard HK, Johnsen SP: Enforcing centralization for gastric cancer in Denmark. Eur J Surg Oncol 2010;36:S50-S54.

40 Birgisson H, Talback M, Gunnarson U, Pahlman L, Glimelius B: Improved survival in cancer of the colon and rectum in Sweden. Eur J Surg Oncol 2005;31:845-853.
Lymph Node Dissection in Advanced Resectable Gastric Cancer 